International Journal of Modern Physics C, Vol. 0, No. 0 (2000) 000-000

(C) World Scientific Publishing Company

\title{
EFFECTS OF ON- AND OFF-RAMPS IN CELLULAR AUTOMATA MODELS FOR TRAFFIC FLOW
}

\author{
G. Diedrich, L. Santen, A. Schadschneider, and J. Zittartz \\ Institut für Theoretische Physik, Universität zu Köln, \\ D-50937 Köln, Germany
}

Received October 31, 2018

Revised

\begin{abstract}
We present results on the modeling of on- and off-ramps in cellular automata for traffic flow, especially the Nagel-Schreckenberg model. We study two different types of onramps that cause qualitatively the same effects. In a certain density regime $\rho_{\text {low }}<$ $\rho<\rho_{\text {high }}$ one observes plateau formation in the fundamental diagram. The plateau value depends on the input-rate of cars at the on-ramp. The on-ramp acts as a local perturbation that separates the system into two regimes: A regime of free flow and another one where only jammed states exist. This phase separation is the reason for the plateau formation and implies a behaviour analogous to that of stationary defects. This analogy allows to perform very fast simulations of complex traffic networks with a large number of on- and off-ramps because one can parametrise on-ramps in an exceedingly easy way.
\end{abstract}

\section{Introduction}

An effective management of transport problems is of great interest in densely populated areas. Therefore the performance optimisation of the existing traffic networks is an important aspect of infra-structural planning 6 . Recent experimental 2 目蚫国 and theoretical studies 6 . mance is largely dominated by the capacity of so-called bottlenecks, i.e. parts of the roads where the capacity is locally reduced. In realistic traffic systems a large variety of possible bottlenecks exists, e.g. road constructions, crossings and lanereductions. If one concentrates on highway networks the reduction of the capacity is often due to on- and off-ramps. Therefore the influence of on- and off-ramps has been discussed, e.g., on- and off-ramps have been used in studies of macroscopic models in order to explain the emergence of synchronised traffic 6 .

In this work we present a simulation study of op-and off-ramps using a discrete

microscopic traffic model 10.11 (for reviews, see 12, 13.14). In order to extract the effects of on- and off-ramps we keep the lattice geometry as simple as possible, i.e. we study a single-lane system with periodic boundary conditions, with an additional on- and off-ramp. Moreover we consider only one type of cars. This choice of the system allows for an easy parametrisation and systematic analysis of ramp effects. 
Nevertheless these simplifications do not reduce the practical relevance of our results because the empirical results which have been obtained at multi-lane highways show the large influence of the ramps 3.9 . Moreover the strong coupling of the lanes at high densities implies that the generic behaviour is found on all lanes 25.61 .17 .

The simulation results show that the maximal capacity of the system depends on the flow at the ramps as well as on different input strategies. In contrast, different output procedures do not affect the results significantly. Starting from this realistic scenario we discuss the relation to periodic systems with stationary defects 18,29 , i.e. to periodic systems where the mobility of the cars is locally reduced. It turns out that the essential features of traffic systems near ramps are already reproduced by the system with a stationary defect. Moreover we are able to establish a direct relation between on-ramp activity and parametrisation of the defect. Therefore it seems to be possible to achieve a considerable reduction of the computational complexity of network simulation using this kind of implementation of ramps.

The influence of on- and off-ramps on the dynamics in microscopic models is not yet analysed to our knowledge. The most important point that is investigated here is the strategy of the cars to change from the acceleration lane to the driving lane. This strategy depends on the individual behaviour of the drivers and might have a considerable influence on the traffic dynamics. In this paper we will present a simple scenario that is able to quantify the effects of on- and off-ramps qualitatively. It will be no restriction to the problem that we will only treat one-lane models. The effects caused by the on-ramps in realistic systems can be explained qualitatively with one-lane models. Therefore the Nagel-Schreckenberg (NaSch) model serves as the basis model for the implementation of on- and off-ramps. Firstly we suggest two different types of on-ramps. It is found that it is not necessary to make a difference in the modelling of the off-ramps because they play a rather inferior role for the traffic dynamics. Afterwards we study the effect of both types on the model qualitatively with the help of suitable observables. It will be shown that there exists a strict analogy of the effect of on-ramps to that of stationary defects. This analogy allows the parametrisation of on-ramps in an exceedingly easy way, so that one can perform very fast, but realistic, simulations of complex traffic networks.

Before we start with our considerations concerning the on- and off-ramps, let us remind the reader of the update rules (parallel dynamics) in the NaSch model:

$$
\begin{array}{ll}
\text { R1 Acceleration: } & v_{i} \rightarrow \min \left(v_{\max }, v_{i}+1\right) \\
\text { R2 Braking: } & v_{i} \rightarrow \min \left(d_{i}, v_{i}\right) \\
\text { R3 Randomization: } & v_{i} \rightarrow \max \left(0, v_{i}-1\right) \\
& \quad \text { with probability } p \\
\text { R4 Driving: } & \text { car } i \text { moves } v_{i} \text { cells }
\end{array}
$$

Here $v_{i}$ and $d_{i}$ denotes the velocity of car $i$ and the number of empty cells in front 
of car $i$, i.e. the so-called headway, respectively. The maximum velocity and the slowdown parameter are denoted as $v_{\max }$ and $p$ respectively.

\section{Definition of the ramp-types}

In this section we discuss two possible types of implementations of on- and offramps. The on- and off-ramps are implemented as connected parts of the lattice where the vehicles may enter or leave the system. The activity of the ramps is characterised by the number of entering (or leaving) cars per unit of time $j_{\text {in }}\left(j_{\text {out }}\right)$. In order to avoid density fluctuations we only added a car to the system if the removal of another car at the off-ramp is possible at the same time-step. So, the following relation for the rates holds: $j_{\text {in }}=j_{\text {out }}$. Moreover, input and output are performed with a constant frequency. Compared to a stochastic in- and output of cars this particular choice allows for a better quantitative analysis of the results. We have tested that a stochastic in- and output of cars does not lead to a qualitatively different behaviour of the system. The chosen length of the on- and off-ramps and the distance between them is motivated by the dimensions found on german highways. Here we have chosen $L_{r a m p}=25$ as length of the ramps in units of the lattice constant (usually identified with 7.5 meters). The position, i.e. the first cell of the on-ramp, is located at $x_{o n}=80$ and that of the off-ramp at $x_{o f f}=L-80$ where $L$ is the system size. Using periodic boundary conditions the distance of the on-ramp to the off-ramp is given by $d_{\text {ramp }}=x_{o n}-x_{o f f}+L$. The only and essential difference in the implementation of the different types of on-ramps lies in the strategy of the cars changing from the acceleration lane to the driving lane.

Now we discribe two different procedures adding cars to the lattice.

\section{Type A}

Using this method the lattice will successively be searched in the region of the onramp $\left(x_{o n}\right.$ to $\left.x_{o n}+L_{r a m p}\right)$ until a vacant cell is found. Then a car will be inserted into this cell (see Fig. 1), even if the cell in front is already occupied by a car. The velocity of the car is set to $v_{\max }$. Dependent on the global density $\rho$ this can lead to a strong perturbation of the system (see Section).

This extremely restrictive method described above implies a very inconsiderate behaviour of the drivers because in the process of changing from the acceleration lane to the driving lane no safety margin will be held. However, due to the unbounded deceleration capabilities of the cars in the NaSch model this will not lead to accidents. 


\section{Type B}

In this method the cell that will be occupied by a car is selected in a stochastic way. A vacant cell will be randomly chosen in the region of the on-ramp. Afterwards a car will be inserted into this cell like in type A. The measurements in the simulations will show that there is no qualitative difference of this type to type A. Type B will also lead to a strong perturbation of the system.

By the method described above one gets a more realistic description of traffic flow than in type A. Here it will be used to simulate both inconsiderate and cautious behaviour of the drivers.

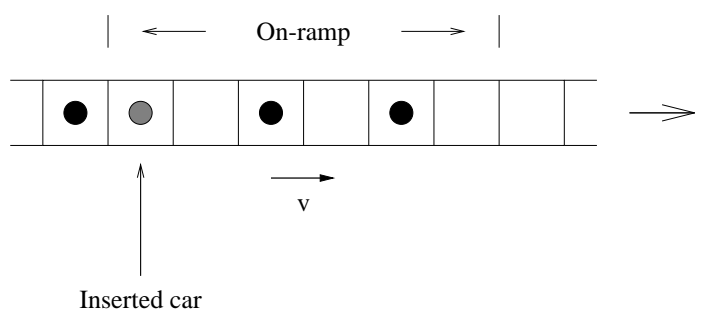

Figure 1: Schematical representation of the on-ramp (Type A).

The off-ramps work for the corresponding types of on-ramps in the same way: one goes successively through the cells of the lattice in the region of the off-ramps until an occupied cell is found. Then the car will be removed from this cell. A schematic diagram of this procedure is shown in Fig. 2.

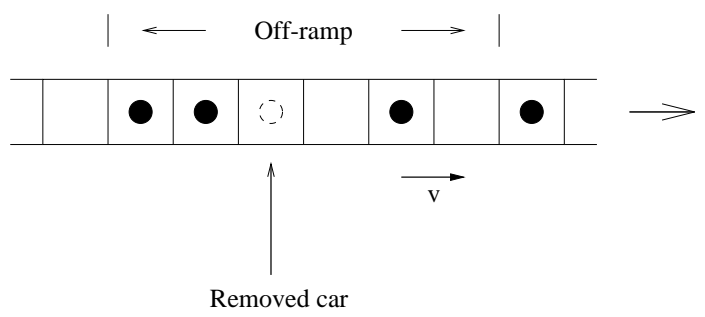

Figure 2: Schematical representation of the off-ramp.

\section{Effects of the ramps}

In this section we discuss the effects of the ramps. Using computer simulations fundamental diagrams dependent on the in/output-rates and density profiles dependent on the global density of the system are generated. Then, by analysing these results, we investigate the relation between on-ramps and stationary defects. This will be done in the last subsection. 


\subsection{Fundamental diagrams and density profiles}

In Fig. 3 the fundamental diagrams of the NaSch model for both types of on-ramps are shown. The input-rate is chosen to be $j_{\text {in }}=j_{\text {out }}=\frac{1}{5}$. This choice guarantees the conservation of the global density $\rho$. For comparison the fundamental diagram without ramps is also shown as solid line.

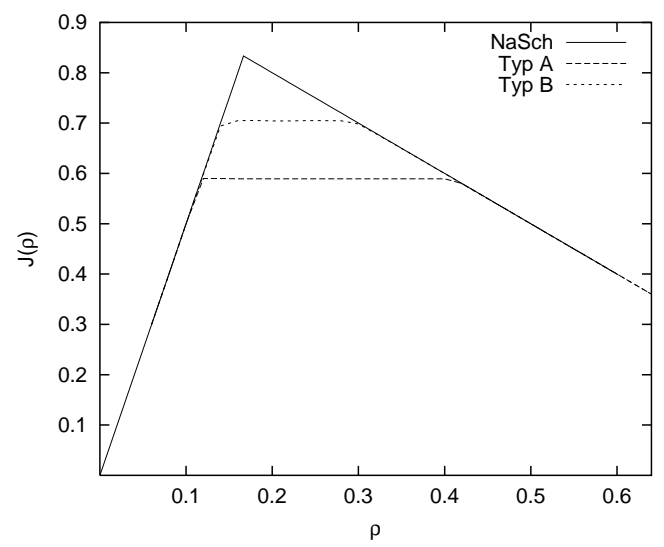

Figure 3: Fundamental diagrams of both ramp types at the same input-rate $j_{\text {in }}=\frac{1}{5}$. The model parameters are given by $L=3000, v_{\max }=5, p=0$.

It is clearly seen that a density regime $\rho_{\text {low }}<\rho<\rho_{\text {high }}$ exists where the flow $J(\rho)$ is independent of the density. This so-called plateau value of the flow is lower than the corresponding flow of the model without ramps. An increase of the input-rate $j_{\text {in }}$ leads to a decrease of the plateau value If one compares the different types of input strategies it is evident that the plateau value of type B is lower than that of type A. This can be explained by the probability that the cell in front of the inserted car is already occupied. For type B this probability is smaller than for type A. Nevertheless there is no difference between the two types in a qualitative sense. That is the reason why we restrict ourselves to type A in the further treatment. With this type the effect can be seen in the simplest way and so it is easier to describe.

Obviously we can distinguish three different phases depending on the global density. In the high and low density phases the average flow $J(\rho)$ of the perturbated system takes the same value as in the system without ramps. For intermediate densities $\rho_{\text {low }}<\rho<\rho_{\text {high }}$ the flow is constant and limited by the capacity of the ramps.

For a better understanding of the behaviour of the average flow it will be helpful to look at the density profile (see Fig. 4). In the high and low density phase one only observes local deviations from a constant density profile. For intermediate densities the system is separated into macroscopic high $\left(\rho_{\text {high }}\right)$ and low $\left(\rho_{\text {low }}\right)$ density regions. In the region of the ramps the density $\rho_{\text {ramp }}$ is additionally higher than in the high 
density region. Thus the ramps act like a blockage in the system that decreases the flow locally. Varying the global density within the phase-separated state the bulk densities in the high and low density region remain constant, only the length of the regions changes (see Fig. 5). Also the local density at the ramps $\left(\rho_{\text {ramp }}\right)$ remains constant. Finally this leads to a constant flow in the segregated phase.

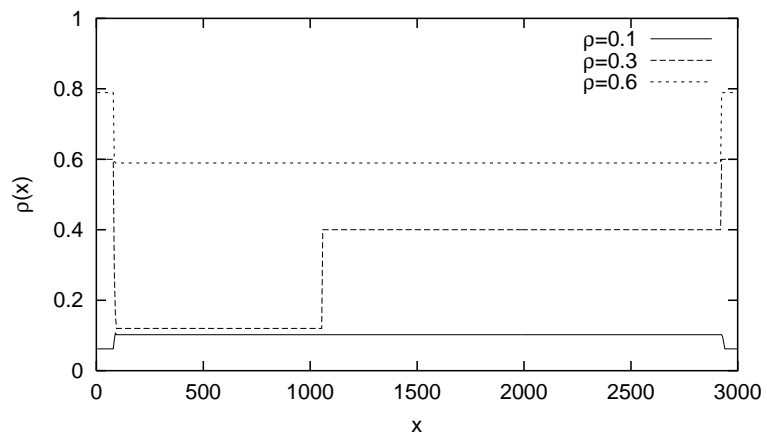

Figure 4: Density profiles in the three phases. In the high $(\rho=0.60)$ and low $(\rho=0.10)$ density phases only local inhomogeneities occur near the ramps $\left(x_{o n}=\right.$ $\left.80, x_{o f f}=L-80\right)$. For intermediate densities $(\rho=0.30)$ one observes phase separation. The model parameters are the same we used in Fig. 3.

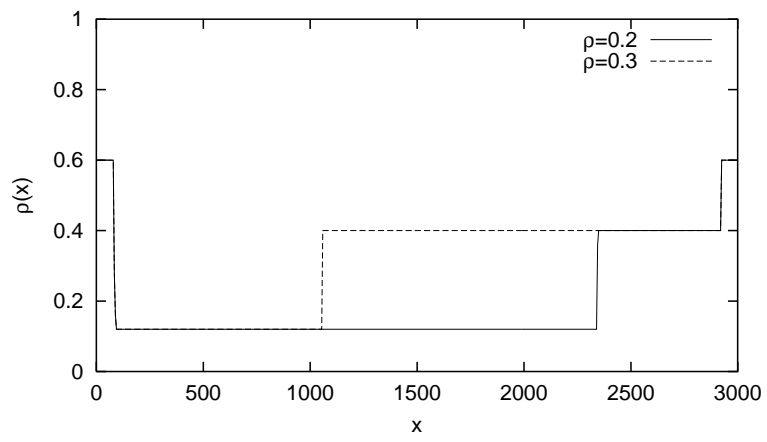

Figure 5: Density profiles for two different densities within the phase-separated state. $\rho_{\text {ramp }}, \rho_{\text {low }}, \rho_{\text {high }}$ remain constant, only the length of the regions changes.

If one assumes that the cars can move freely in the low density regime and that the flow in the high density region depends linearly on the density (which holds for small braking parameters $p$ ) we get the following relation:

$$
\rho_{\text {low }}=\left(1-\rho_{\text {high }}\right) \frac{1-p}{v_{\max }-p} .
$$

Unfortunately no expressions for $\rho_{\text {low }}$ and $\rho_{\text {high }}$ depending on the model parameters 
only can be given. The reason is that no exact analytical description for the unperturbated system with $v_{\max }>1$ has been found so far. Furthermore an analytical treatment of the ramps is very complicated because of the strong interactions within these regions.

\subsection{Analogy to stationary defects}

In the previous subsection we have shown that the on-ramps in the system act like a blockage. This blockage leads to a decrease of the local flow at the ramp. To clarify the analogy of on-ramps to stationary defects we give a short summary of the most important results on stationary defects like construction sites 18.19.

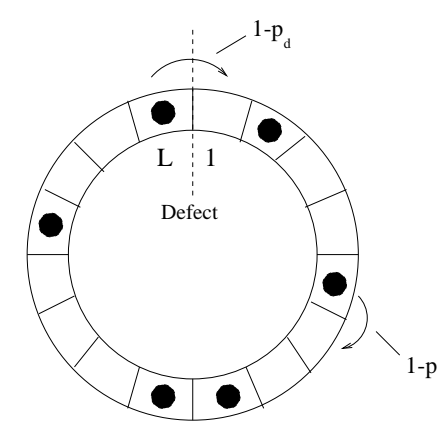

Figure 6: Schematical representation of a single defect in the NaSch model with $v_{\max }=1$. In this special case the average velocity of the cars is approximately $v=1-p$ for $\rho \ll \frac{1}{2}$. At the defect site the average velocity is $v=1-p_{d}$.

Stationary defects can be implemented in two different ways. First, one can define a certain range on the lattice where the slowdown parameter $p$ is increased compared to that of the residual lattice sites 19 Second, one can decrease the maximum velocity $v_{\max }$ in this certain range 20.21. In our investigations we restrict ourselves to the first method.

Fig. 6 shows a schematical representation of a single defect in the NaSch model with $v_{\max }=1$ and slowdown parameter $p$. The slowdown parameter at the defect is denoted as $p_{d}$.

In Fig. 7 the fundamental diagram of the NaSch model with stationary defects is represented. Again we can distinguish three different phases. In the high and low density regime the flow $J(\rho)$ is identical to that of the model without defects. For intermediate densities $\rho_{\text {low }}<\rho<\rho_{\text {high }}$ the flow is constant in analogy to the model with ramps. In this regime $J(\rho)$ is limited by the capacity of the defect sites.

Once again the phenomenon of the plateau formation can be understood by the analysis of the density profiles (see Fig. 8). The argument is the same as in subsection 3.1.

For the case $v_{\max }=1$ of the NaSch model with a single defect site good approxi- 


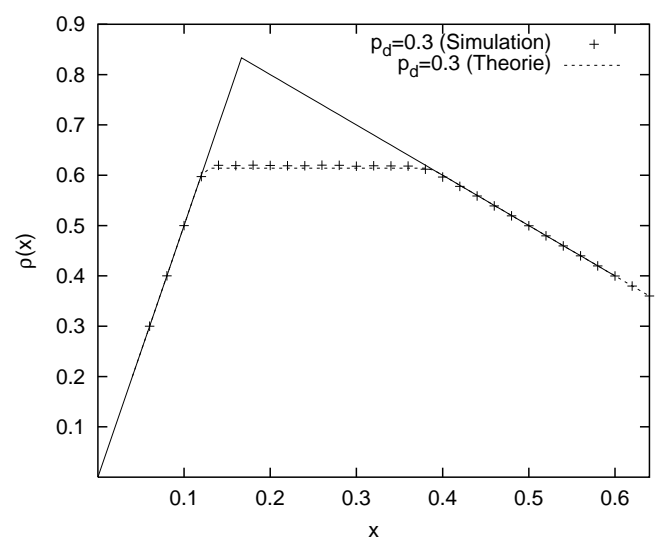

Figure 7: Fundamental diagram of the NaSch model with stationary defects. The model parameters are given by $L=3000, p=0, v_{\max }=5$. For comparison the diagram of the model without defects is shown as solid line.

mations for the plateau value of the flow as well as for the densities $\rho_{l o w}$ and $\rho_{\text {high }}$ can be made 19. An essential reason for this is the particle-hole symmetry in this special case. Because of this symmetry following relation holds:

$$
\rho_{\text {low }}+\rho_{\text {high }}=1
$$

With the assumption that the system is separated into two regions of constant density and that the flow $J_{d}$ at the defect is equal to the flow $J_{b u l k}$ in the bulk one can establish following equation in the mean field theory:

$$
J_{d}=q_{d} \rho_{\text {high }}\left(1-\rho_{\text {low }}\right)=J_{\text {bulk }}=q \rho_{\text {high }}\left(1-\rho_{\text {high }}\right)
$$

where $q=1-p$ and $q_{d}=1-p_{d}$. With equation (3.2) we finally get the expressions for the densities:

$$
\rho_{\text {high }}=\frac{q}{q+q_{d}} \quad, \quad \rho_{\text {low }}=\frac{q_{d}}{q+q_{d}} .
$$

From this and the exact current (see e.g. 11.22) follows the plateau value of the flow:

$$
J_{P}=\frac{1}{2}\left(1-\frac{1}{q+q_{d}} \sqrt{\left(q+q_{d}\right)^{2}-4 q^{2} q_{d}}\right) .
$$

In principle one can treat the defects for $v_{\max }>1$ in the same way. When we make the assumption that for the two branches in the fundamental diagram the following approximations hold:

$$
J_{\text {free }}=\rho\left(v_{\max }-p\right) \quad, \quad J_{\text {jam }}=(1-p)(1-\rho),
$$




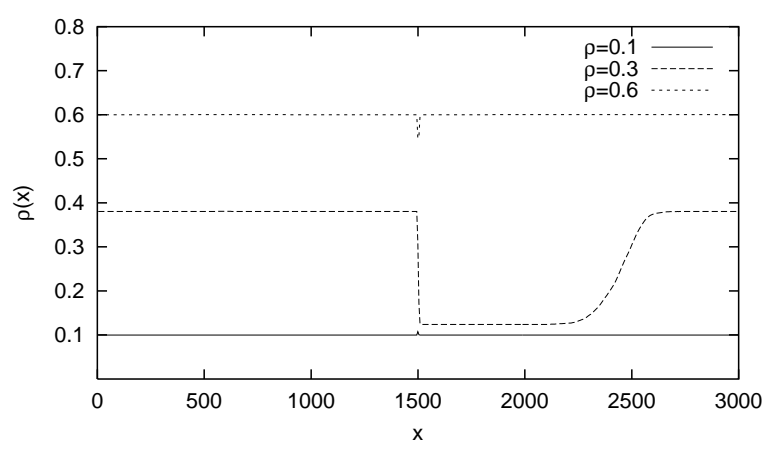

Figure 8: Density profiles of the NaSch model with stationary defects for densities in the three phases. In the high $(\rho=0.60)$ and low $(\rho=0.10)$ density phases only local inhomogeneities occur near the defect sites $\left(L-v_{\max } \leq x_{d}<L\right)$. For densities within the plateau regime one observes phase separation.

one gets the following expressions for the densities in the phase-separated state:

$$
\begin{aligned}
\rho_{\text {low }} & =\frac{q_{d}}{v_{\max }-p+q_{d}}, \\
\rho_{\text {high }} & =\frac{\left(v_{\max }-p\right)\left(q-q_{d}\right)+q q_{d}}{\left(v_{\max }-p\right) q+q q_{d}} .
\end{aligned}
$$

The plateau value of the flow results from this as:

$$
J_{P}=\frac{\left(v_{\max }-p\right) q_{d}}{v_{\max }-p+q_{d}} .
$$

In spite of the crude estimate for $J_{j a m}$ this approximation is in good agreement with the simulation data for small $p$ (see Fig. 7). It has not been checked so far for which range of values for $p_{d}$ equation (3.8) can reproduce the simulation results. We have shown that there is no qualitative difference between the effect of onand off-ramps and that of stationary defects. In both cases one observes plateau formation in the fundamental diagram as well as phase separation in the system. The only difference lies in the nature of the blockage dividing the system into two macroscopic regions. In the case of the ramps it is the local increase of the density that decreases the flow locally. In the model with defects the increased slowdown parameter leads to a local decrease of the flow.

In order to use the equivalence of ramps and defects in realistic simulations of traffic flow one has to determine the relation between the input-rates $j_{\text {in }}$ and the slowdown parameter $p_{d}$ at the defect sites. Fig. 9 shows the values of $p_{d}$ and $j_{\text {in }}$ leading to the same fundamental diagrams. It can be seen that a non-trivial relation between the two parameters exists. We regard this Fig. 9 as our main result for future applications. 


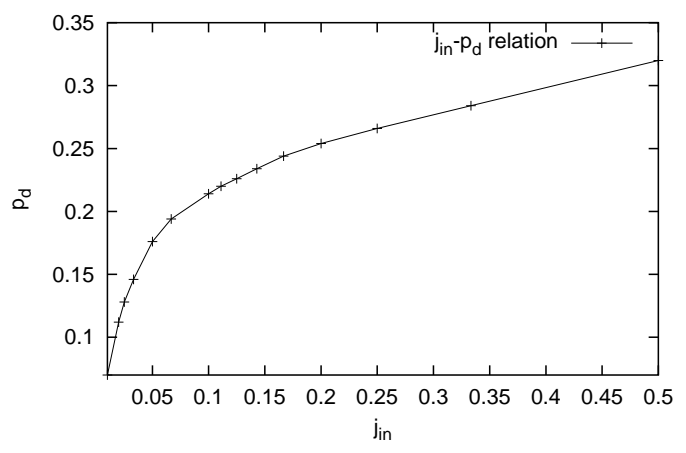

Figure 9: Relation between the input-rates $j_{i n}$ and the slowdown parameter $p_{d}$ at the defect sites.

\section{Summary and Conclusions}

In this paper we have presented a simple scenario that can quantify the effects of ramps on the traffic dynamics in microscopic models. The model used here is the well known Nagel-Schreckenberg model, a cellular automaton for traffic flow. We have introduced two different types of on-ramps. One type that implies a very inconsiderate behaviour of the drivers and another type that simulates both inconsiderate and cautious behaviour. We have shown that there is no difference between these two types in a qualitative sense. With both types one observes effects very similar to those of stationary effects: in both cases one observes plateau formation in the fundamental diagram as well as phase separation in the system. The only difference lies in the mechanism dividing the system into two macroscopic regions. This analogy is is very important for efficient modelling of complex networks 1 with a multitude of ramps.

Usually in simulations of large networks one uses a more realistic approach. In 23 ramps are fed by an external road. Cars try to change to the highway according to the usual lane changing rules. If they could not change the cars wait at the end of the ramp.

In our considerations we have also investigated in the influence of ramps on different mutations of the NaSch model like the Slow To Start model 2425 and a model with an anticipation rule. The effects caused by the ramps are qualitatively the same as in the NaSch model, but in the model with anticipation one has to use much higher input-rates $j_{\text {in }}$ to observe phase separation and plateau formation. This model reacts more robust on the perturbations caused by the cars changing from the acceleration lane to the driving lane 26.

The effects of ramps have been studied in connection with synchronised traffic recently 6 . For the hydrodynamic models it has been found that the localised perturbation through the ramp flow can induce transitions between different traffic 
sta.tes.

In 9 it has been shown using computer simulations and data from measurements on real traffic that on-ramps can induce a first-order non-equilibrium phase transition between free flow and a congested phase. The transition is induced by the interplay of density waves caused by the on-ramps and shock waves moving on the highway.

\section{Acknowledgment}

This work has been performed within the research program of the SFB 341 (KölnAachen-Jülich). L. S. acknowledges support from the Deutsche Forschungsgemeinschaft under Grant No. SA864/1-1.

\section{References}

1. K. Nagel, J. Esser and M. Rickert, in: Annu. Rev. Comp. Phys., ed. D. Stauffer (World Scientific, 1999)

2. C.F. Daganzo, M.J. Cassidy, R.L. Bertini, Transp. Res. A 33, 365 (1999)

3. B.S. Kerner, H. Rehborn, Phys. Rev. E 53, R4275 (1996)

4. B.S. Kerner, H. Rehborn, Phys. Rev. Lett. 79, 4030 (1998)

5. B.S. Kerner, Phys. Rev. Lett. 81, 3797 (1998)

6. L. Neubert, L. Santen, A. Schadschneider, M. Schreckenberg, Phys. Rev. E 60, 6480 (1999)

7. H.Y. Lee, H.-W. Lee, D. Kim, Phys. Rev. Lett. 81, 1130 (1998); Phys. Rev. E 59, 5101 (1999)

8. D. Helbing, M. Treiber, Phys. Rev. Lett. 81, 3042 (1998)

9. V. Popkov, L. Santen, A. Schadschneider, G. Schütz, preprint (2000)

10. K. Nagel and M. Schreckenberg, J. Physique I, 2, 2221 (1992)

11. M. Schreckenberg, A. Schadschneider, K. Nagel and N. Ito, Phys. Rev. E 51, 2939 (1995)

12. Wolf D.E., Schreckenberg M., Bachem A. (Eds.): Traffic and Granular Flow (World Scientific, Singapore 1996)

13. Schreckenberg M., Wolf D.E. (Eds.): Traffic and Granular Flow '97 (Springer 1998)

14. Chowdhury D., Santen L., Schadschneider A., Phys. Reports (in press)

15. D. Chowdhury, D. E. Wolf, M. Schreckenberg, Physica A 235 , 417 (1997)

16. W. Knospe, L. Santen, A. Schadschneider, M. Schreckenberg, Physica A 265, 614 (1999)

17. D. Helbing, B.A. Huberman, Nature 396, 738 (1998)

18. S.A. Janowsky, J.L. Lebowitz, Phys. Rev. A 45, 618 (1992); J. Stat. Phys. 77, 35 (1994)

19. W. Knospe, L. Santen, A. Schadschneider, M. Schreckenberg, in 13, p. 349

20. Emmerich H., Rank E., Physica A216, 435 (1995)

21. Csahok Z., Vicsek T., J. Phys. A27, L591 (1994)

22. A. Schadschneider, Eur. Phys. J. B 10, 573 (1999)

23. Rickert M.: Master Thesis, Universität zu Köln (1994)

24. A. Schadschneider and M. Schreckenberg, Ann. der Phys. 6, 541 (1997)

25. R. Barlovic, L. Santen, A. Schadschneider and M. Schreckenberg, Eur. Phys. J. 5, 793 (1998)

26. Diedrich G.: Numerische Untersuchung zur Phasenseparation in Zellularautomaten für den Strassenverkehr, Masters Thesis, Universität zu Köln (1999) 